\title{
Scaffold Free 3D Culture of Mesenchymal Stem Cells; Implications for Regenerative Medicine
}

\begin{abstract}
Keywords: Mesenchymal stem cells; Three dimensional cell culture; Regenerative medic ine

Abstract

Mesenchymal stem cells (MSCS) are recognized as important sources for regenerative medicine (RM). Adherent cultures, long time considered definitory for MSCs phenotype, are relatively easy to perform and to assess. Two dimensional cell expansion systems, however, have been shown to decrease stemness and to induce cellularsenescence therefore impeding on MSC therapeutic potential.

Multic ellular aggregates known as spheroids are a form of three dimensional (3D) culture increasingly recognized to improve MSC differentiation potential to mesenchymal and non-mesenchymal lineages, to increase cytokine and growth factor release as well as cell trafficking and survival after transplantation. Data about improved vasculogenesis and tissue regeneration potential of spheroid cultured MSCs are already available, making this method attractive for regenerative strategies in wound healing, revascularization after myocardial and cerebral infarctation or peripheral ischemia. In-depth genetic and epigenetic profiling of MSCs in this particular state would be needed in order to predict their therapeutic potential for RM. Better understanding of changes in cellular phenotype that occurs in spontaneous 3D and the implication for in vivo cell behavior as well as cell host interaction after transplantation are required before clinical applications.
\end{abstract}

\section{Introduction}

Mesenchymal stromal or stem cells (MSCs) are phenotypically heterogenous populations of adult progenitors, initially isolated from the stroma of bone marrow by Friedenstein and coworkers [13]. The team described a population of fibroblast-like cells capable of forming colonies and of differentiating in vitro to osteocytes. Further studies identified a multitude of mesenchymal tissues that contain and can be used as sources of MSCs such as adipose [4] skeletal muscle [5] trabecular bone [6], dental pulp [7], cord blood [8]. MSCs are capable of differentiating into specific mesenchymal and non-mesenchymal lineages. Bone, cartilage, muscle, bone marrow stroma, tendon/ligament, adipose, dermis, and other connective tissues $[9,10]$ and as well, [11] glia [12], hepatocytes [13] or endothelial cells $[14,15]$ could be obtained from MSCs in defined culture conditions in vitro as well as in vivo in various animal models. Due to their ability to generate tissue-specific cells as well as to secrete trophic factors and elicit immune modulation, MSCs are sought as cell sources for regenerative medicine (RM). International Society for Cell Therapy (ISCT) has identified the minimal criteria for MSCs characterization among which the capability to adhere to the culture dish is considered a functional definition of phenotype [16]. Recent reports about improved proliferative and differentiation potential of MSCs of various tissue sources in three dimensional (3-D) culture

\section{Journal of}

\section{Transplantation \& Stem Cell Biology}

\author{
Luminita Simion Labusca ${ }^{1,2 *}$ \\ ${ }^{1}$ Department of Orthopedic Surgery, University Hospital Saint \\ Spiridon Iasi Romania, Romania \\ ${ }^{2}$ Department of Clinical Veterinary and Animal Science, Faculty of \\ Health and Medical Sciences, University of Copenhagen, Denmark
}

Address for Correspondence

Luminita Simion Labusca, Department of Orthopedic Surgery, University Hospital Saint Spiridon lasi Romania, Bd Independentei nr 1, cod 700111 IASI, Romania, Tel: +40232240822; E-mail: drlluminita@yahoo.com

Submission: 29 July 2015

Accepted: 05 September 2015

Published: 10 September 2015

Copyright: (c) 2015 Labusca LS. This is an open access article distributed under the Creative Commons Attribution License, which permits unrestricted use, distribution, and reproduction in any medium, provided the original work is properly cited.

Reviewed \& Approved by: Dr. Yong Li, Associate Professor, Department of Pediatric Surgery, University of Texas, USA

systems $[17,18]$ invites to revisiting the definition of mesenchymal progenitors as adherent populations. This review will describe several features of scaffold free 3-D culture that recommend this approach as a tool for improving understanding of MSC biology as well as for generating successful RM therapies.

\section{D Versus 3D Cultures of MSCs}

With the classical approach, a mononuclear cell suspension derived from the donor tissue by mechanical separation, gradient centrifugation or enzymatic digestion is plated at a density of about $1.6 \times 10^{5} \mathrm{cells} / \mathrm{cm}^{2}$ in flasks with stiff and flat polystyrene culture surface. Initial cell suspension contain variable amounts of MSCs ranging from $0.0001 \%$ in the bone marrow to almost $0.1 \%$ in the stromal vascular fraction (SVF) resulting from adipose tissue processing [19]. Due to their paucity in the tissue of origin, for research but most of all for therapeutic purposes, MSCs need to be expanded by means of successive subcultures (passages), an operation that involves detaching the cells from the culture surface mostly by enzymatic digestion. The regular culture media contains a basal formulation (Dulbecco's modified Eagle's medium or minimal essential medium) in the presence of fetal bovine serum (FBS) [20]. Used for several decades, this technique is considered relatively simple and highly reproducible, enabling cell sorting based on their capability to adhere to plastic. It results in relatively homogenous MSC populations and allows for simple straightforward gestures for maintenance such as media change or microscopic evaluation. Culture automation and standardization amendable for large scale production of MSCs is facilitated using this approach [21], this enables the development of commercially available solution for therapeutic applications [22]. Moreover, cells imaging as well as assessment of differentiation in flat culture can be done using regular inverted (fluorescent) microscopes, in most cases without the need of embedment techniques and slide preparation required for 3D structures.

However, several technical as well as biological problems arise 
with the use of conventional flat culture surfaces. From a technical perspective, the dependence on FBS for cell expansion introduces both the hazard of using xenogeneic proteins as well as batch variability of the product known to influence MSCs proliferation and survival [23]. This introduces the necessity that each batch should be tested for the suitability of being used for MSC culture. Moreover, increased efforts are made to introduce xenofree media for manufacturing MSC for therapeutic purposes. Human platelet lysate, pooled human plasma or recombinant cytokines are tested as culture supplements to avoid potential hazards from the use of animal derivatives. From a biological perspective, monolayer culture introduces an artificial environment for adhesion, feeding and motility that alters cellular phenotype and behavior. In normal conditions, within a tissue, cells are embedded within an extracellular matrix (ECM). This situation implies three dimensional cells-cell and cell-ECM contact and communication, as well as a specific cell polarity, access to the interstitial fluid containing nutrients and signaling molecules and context dependent motility. On a flat culture surface, approximately half of the cell surface is exposed to culture media and the other half to the substrate of the culture dish, a low percent interacts with other cells [24]. As a consequence, MSCs expanded in monolayer lose their capability to proliferate, differentiate and forming colonies after a variable amount of passages [25] are exposed to culture media induced replicative senescence and reduced multipotency [26]. Such challenges impede equally on enhancing knowledge about stem cell behavior while in vivo as well as on the design of safe and efficient regenerative therapies.

The necessity of adding a third dimension to cell culture system that more closely resembles the natural environment and might generate significant differences in MSCs phenotype, behavior and therapeutic potential is increasingly recognized in the literature [27]. The advantages of $3 \mathrm{D}$ cultures in terms of reproducing intercellular communication and in generating complex tissue- like structures has stimulated technical developments. Various scaffolds and coating materials that add a dimension to the culture surface as well as of bioreactors that facilitate nutrient diffusion within complex constructs are largely used in the field of stem cells for research as well as for translational purposes [28]. Interestingly, the growth of MSCs, known to be anchorage dependent cells in low attachment dishes has been reported to induce the formation of cellular aggregates [29]. Due to their spherical shape, such aggregates are denominated spheroids (Figure 1). To note, the method of culturing multicellular aggregates as spheroids was first described in relation with embryonic cells, established as a method in cancer research [30] and only relatively recent in the field of stem cells [31] and tissue engineering [32].

\section{Technical Approach on Spheroid Culture}

In $2 \mathrm{D}$ culture conditions, adherent cell populations when seeded as a cell suspension in the culture dish would fall due to gravity to the surface of the culture dish and form connections via cell surface integrins with the ECM-like molecules (such as fibronectin) adsorbed on the plastic [33]. Whenever the surface of the culture dish is not permissive for adhesion, the cells would tend to agglomerate in relatively homogenous suspended 3D clusters. The modalities used for inducing or enhancing the formation of spheroids can be static or dynamic. Static method implies providing cells a modality to form

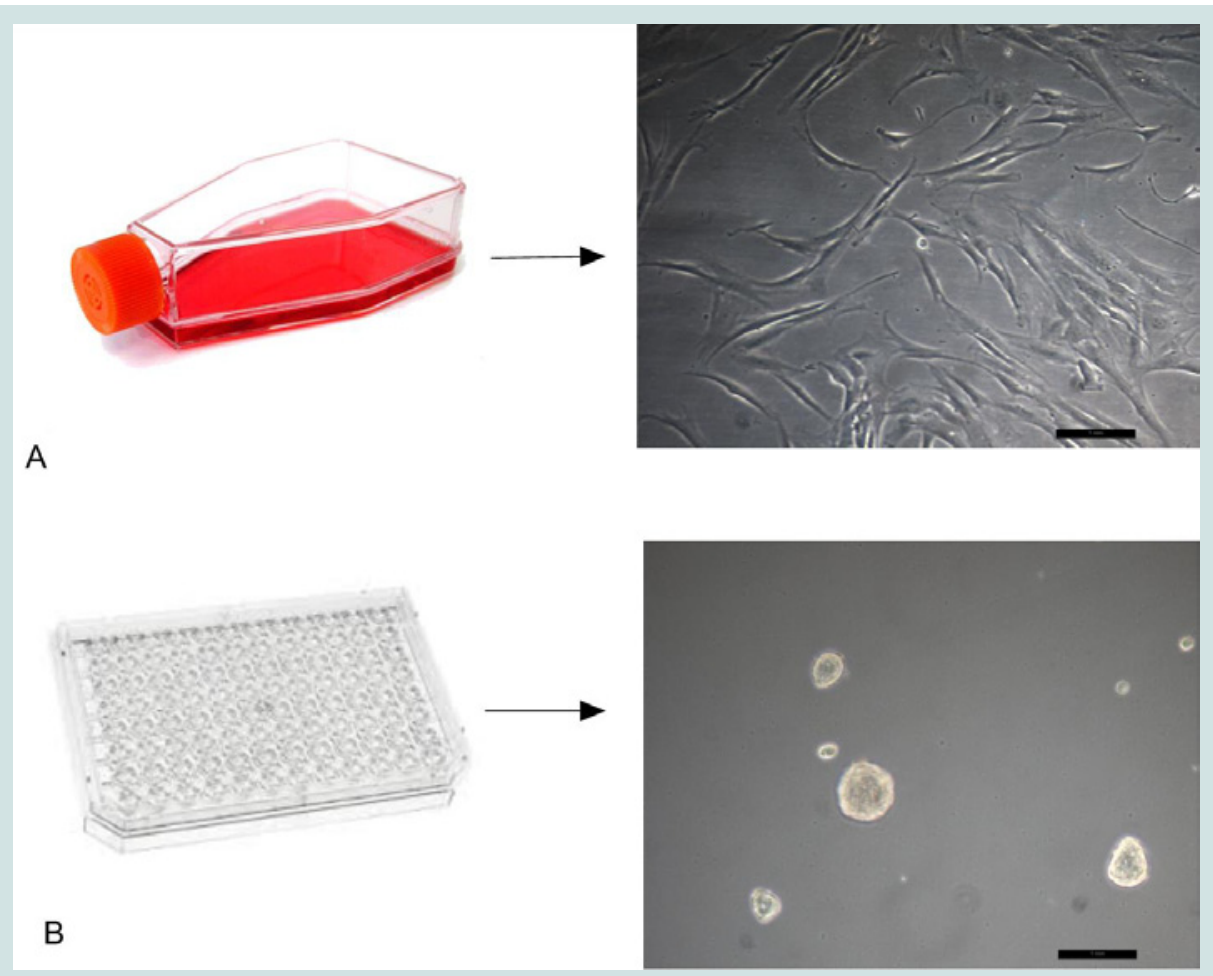

Figure 1: 2D versus 3D culture of same cell population (Adipose derived stem cells passage 3).

(A) "Classical" culture method using polystyrene coated cell culture flask and the microscopic appearance of adherent cell population -scale bar $1 \mathrm{~mm}$. (B) Self aggregation and spheroid formation in low attachment culture dish and serum free culture media supplemented with FGF and EGF-scale bar 1 mm. 
aggregates due to static physical forces, one of the earliest is the hanging drop method [34]. Cells spontaneously aggregate at the bottom of a drop resulting from inverting a plate where small amounts of cell culture media ("drops") have been deposited separately from each other. The liquid overlay technique uses a non-adherent substrate, a thin coating of agar or agarose; this prevents cells from attaching and inducing formation of spheroids. The later has been reported to generate more homogenous and reproducible cellular aggregates in mono culture as well as in co-culture systems when compared to hanging drop and to suspension of cells in carboxymethyl cellulose solution [35].

More recently, for laboratory settings, commercially available plates with low attachment surface allow for spontaneous formation of spheroids in suspension culture. By using multiwell (for example, 96 well) plates, the size of the aggregate can be easily controlled by adjusting the number of cells seeded per well. Micro fluidics-generated water-in-oil-in-water $(\mathrm{w} / \mathrm{o} / \mathrm{w})$ double-emulsion (DE) droplets have been reported to increase control of spheroid size, reduce fabrication time and allow for convenient retrieval of cell aggregates that could be suitable for high throughput experiments as well as for large scale manufacturing purposes [36].

Dynamic methods imply forced cellular aggregation. The spinner flask method uses the intrinsic centripetal force resulting from low to medium speed rotation of the culture dish to enable cell aggregation, to prevent adhesion to the bottom and to facilitate intercellular bonding by promoting cell to cell collision by means of constant stirring [37].

The rotating wall vessel provides an internal microgravity environment maintaining the cells in suspension and allowing spheroid formation in a low shear environment, maintaining the cells in a continuous free fall [38]. Extrinsic applied forces such as centripetal force, electrical or magnetic fields or ultrasound act by concentrating cells in high density facilitating aggregation.
Pellet culture uses the centripetal force to aggregate cells at the bottom of a tube or well. Speed and time for centrifugation varies with tube/well volume, cell type and quantity; the method is commonly used to induce chondrogenetic differentiation of MSCs in high density culture $[39,40]$.

Suspension culture of cells after incubation with ferum oxide nanoparticles uses magnetic force to induce a "levitation" system of cellular aggregation [41]. An ultrasound wave trap has been used, as well as an external force, to concentrate cells and facilitate their aggregation [42] (Figure 2).

Cellular self-assembly has been sought as mimicking natural events that occur during embryogenesis, morphogenesis and organogenesis, processes at least, partially; explained by cell surface tension and cadherin isoforms expression [43,44]. Little is known; however, about the impact of culture-system-induced intrinsic or external forces on cell biology. Given the known MSCs mechanosenstivity in terms of cell survival, proliferation and differentiation [45], the contribution of culture system induced mechanical forces to cell phenotype would need further clarification before such procedures can be translated to clinical applications.

\section{Spheroid Culture in Stem Cell Research}

The culture of cellular aggregates is a widely used method for testing pluripotency in embryonic stem cells (ES) or induced pluripotent stem cells (IPSCs) [38]. Spheroids formed in vitro in suspension cultures by pluripotent cells, denominated embryoid bodies, (EB) are commonly used to induce differentiation into cells of the ectoderm, mesoderm, and endoderm lineages. After enzymatic dissociation, single cell population originating from Ebs cultured in defined media can give rise to progenitors of specific lineages pertaining to all three germ layers [46] EB as a form of sponataneous 3D culture system reproduces to a certain extent the intecellular interactions and molecular pathway cascades activated during early

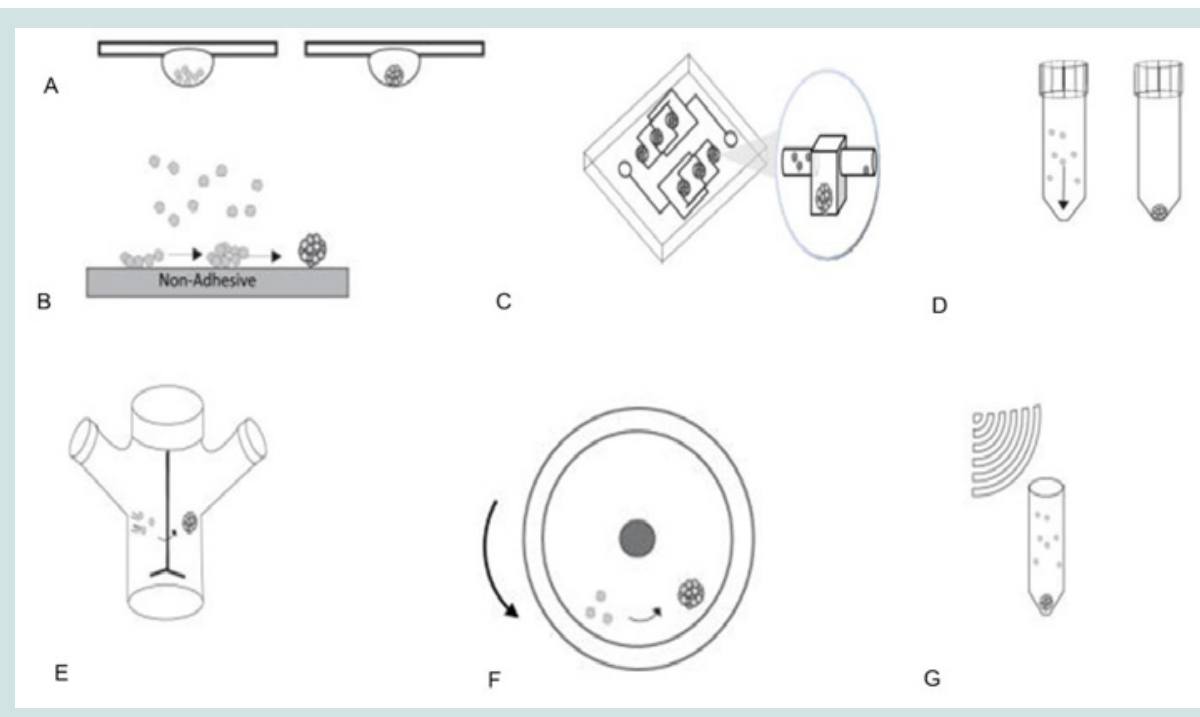

Figure 2: Schematic representation of several technical methods used for obtaining spheroid cultures.

"Hanging drop" technique (B) Liquid overlay (C) Microfluidics system (D) Pellet culture (E) Spinner flasks (F) Rotating vessels (G) Application of external forces (adapted from Ref [38]). 
embryogenesis [47]. Moreover, spheroid culture of other pluripotent or multipotents cell types such as neural stem cells (NSCs) [48], mamary stem cells [49] or corneal stem cells [50] have been used as a method for isolation, in vitro expansion and differentiation to their specific progenies. Stem cells with self renewal capabilites are usually prone to forming spheroids in non-adherent culture conditions in vitro, displaying increased viability and differentiation capabilities [51]. Cells grown as spheroids have been reported to display differentiation abilities not obtainable in 2D systems. Thus, bone marrow MSCs in aggregate culture could be differentiated to hepatocyte-like cells [52], salivary-gland-derived progenitors could be induced to pancreatic islets and hepatocyte lineage [53]. Interestingly, suspended serum-free culture of mouse EB in defined media was able to generate specific neural lineage (telencephalic precursors) directed by specific small molecules added to the culture media [54]. Remarkably, even complex organs such as optic cup could be derived in similar conditions in vitro. Autonomous formation of the retinal primordium structure from mouse 3D ES spheroid culture was reported. Retinal epithelium spontaneously formed hemispherical epithelial vesicles that became patterned along their proximal-distal axis and was able to derive stratified neural retinal tissue $[55,56]$. Human IPSCs cultured as spheroids in defined serum-free media were able to generate neurons and glial cells organized in laminated cortical structure showing transcriptional similarities with human fetal neural cells. Such cells were proven to be electrophysiologically mature and able to form functional networks and to produce synaptic events [57]. It has been proposed that multicellular aggregates of stem cells form complex systems that can self-organize in a similar manner to naturally occuring organogenesis. Deciphering what has been denominated „cytosystem dynamics” could lead to enhanced knowledge about embryonic development, tissue formation and architecture empowering novel strategies for tissue engineering and RM [58].

\section{Particularities of MSCs Spheroids}

MSCs are able to self-aggregate forming spheroid structures with distinctive cell morphology, functionality as well as transcriptional profile as compared to their $2 \mathrm{D}$-cultured counterparts.

When suspended in non-adherent cultures, MSCs initially form cadherin-dependent loose bonds [59] followed by cytoskeleton actomyosin-dependent contraction of aggregates [60]. Cell size is smaller, cell situated inside the aggregate are round while those from the outer layer cells have a more elongated shape, with an overall decrease of cytoskeletal molecules [61]. Cells within spheroids are interconnected with other cells and ECM molecules. Interface connection Young's elasticity modulus is less than $0.1 \mathrm{KPa}$ compared to adherent cultures where the same parameter is in the range of several GPA [62]. Cell size and morphology, cytoskeleton tension as well as the mechanical properties of the substrate are known to influence MSCs differentiation potential [63]. MSCs retrieved from long term spheroid culture "mesenspheres" displayed more robust osteo- and adipogenesis capabilities (matrix mineralization and cytoplasmic lipid accumulation) when compared to MSCs maintained in 2D [64]. Aggregate culture of MSCs was shown to influence cellular phenotype, modifying gene expression profile and inducing epigenetic changes.
There are major differences in gene expression profile of human MSCs in spheroid culture as compared to 2D. DNA microarray revealed up-regulation of genes related to hypoxia, inflammation, stress response including VEGF, cathepsins, angiopoietin2 (ANGPT2), and FGFs, this is possibly explained by the fact that some in the cells from the innercore of the spheres are hypoxic [65]. Calcium-associated genes were found to be more numerous in 3D-cultured MSCs calcium associated receptors 5-hydroxytryptamine receptor 2A and 7 (HTR2A and HTR7) and other intracellular calcium modulators could be implicated in cytoskeleton rearrangement while calcium dependent intracellular signaling molecules such as MAP3k8 (mitogen-activated protein kinase 8) could be implicated in transduction of G-coupled proteins initiating signals that promotes actin reorganization and cell migration. As expected, genes controlling cell-to-cell adhesion were as well found to be up-regulated on MSCs aggregating on chitosan membranes including cadherins, cell adhesion molecules (CAMs), Notch, and cadherin 18 (CDH18). Chemokines and chemokine receptors such as CXC motif chemokine receptor 4 and 7 (CXCR4 and CXCR7), receptors of stromal derived factor-1 (SDF1 or CXCL12) implicated in cell proliferation, migration and differentiation are as well up-regulated in 3D cultured MSCs. Genes associated with cell fate decision, transforming growth factor beta 3 (TGF $\beta 3$ ) bone morphogenetic protein (BMP2), hepatocyte growth factor (HGF) insulin-like growth factor 1 receptor (IGFR1) were as well found to be up-regulated, this possibly explains the enhanced differentiation potential. A higher fold increase of anti-inflammatory interleukins -IL1RN (interleukin 1 receptor antagonist), IL4I1 (interleukin 4 induced gene 1 compared to pro inflammatory ones IL1A (interleukin 1 alpha), IL1B (interleukin 1 beta), IL33 (interleukin 33), and TNFSF13B (tumor necrosis factor ligand super family member 13B) and of Leukemia inhibitor factor (LIF) and its receptor subunit could explain the increased anti-inflammatory as well as antitumor properties of 3D-cultured MSCs [66].

As assessed by RT-PCR, spheroid cultured placental MSCs spheroid hMSCs showed increased mRNA levels of octamer-binding transcription factor 4 (OCT-4) sex determining region Y)-box 2) (Sox-2) homeobox protein Nanog (Nanog) and telomerase reverse transcriptase (TERT) genes, explaining their enhanced clonogenic and proliferation capabilites demonstrated after dissociation [67]. A note of caution needs to be taken into account when interpreting the quantitative results of gene expression analysis performed by $\mathrm{qPCR}$ in experiments involving MSCs spheroids. As cytoskeleton-related gene expression is known to be down-regulated, it is preferable to use a different internal normalize than-actin (ACTB) in order not to overinterpret upregulated genes [33].

Epigenetic profiles were as well found to be modified in $3 \mathrm{D}$ cultures. Increased expression of miR-489, miR-370 and miR-433 is shown to be associated with stem cell pluripotency and differentiation potential [62] as well as increased histone $\mathrm{H} 3 \mathrm{~K} 9$ acetylation levels in the promoter regions of pluripotency genes OCT-4, Sox-2, Nanog and TERT [68].

\section{Spheroid MSCs and their Relevance for Translational Regenerative Medicine}

Due to their biological characteristics, MSCs are considered as a valuable cell source for RM. MSCs differentiation capabilities 
represent the biological basis for the structural effect in recomposing damaged or diseased tissues [69,70]. MSCs have been shown to exert cytokine-mediated paracrine effects contributing to tissue repair by means of the so-called trophic effect on the host and by recruiting local cell populations to induce repair [71]. MSCs are also endowed with immunomodulatory properties capable to restore tissue homeostasis and favor repair [72].

Spheroid culture has been shown in various studies to enhance MSC differentiation potential, cytokine release, angiogenetic effect and tissue repair capabilities enabling the cells to display enhanced stemness and cell survival after implantation [33].

Rat MSC spheroids were shown to display enhanced osteogenesis compared to monolayer cells cultivated in vitro. Enhanced cell survival and in vivo osteogenesis after implantation within a rat model of calvarial defect demonstrated the superior osteoregenerative capabilities of scaffold free 3D-cultured cells [73]. When cultured in vitro in specific differentiation media, adipose-derived MSCs (ADSCs) displayed increased markers of osteogenesis: runt-related transcription factor-2 (runx-2), adipogenesis: lipoproteinlipase (LPL) and peroxisome Proliferator-Activated Receptor Gamma (PPRG) and chondrogenesis (collagen II and aggrecan) [74]. Moreover, ADSCs as floating sphere were proven to transdifferentiate to ectodermal and endodermal lineages [75]. Exposed to media containing hepatocyte growth factor (HGF) fibroblast growth factor (FGF) 1 and 4, floating ADSCs spheres were shown to differentiate to functional hepatocitelike cells capable of producing urea and of storing glycogen that could integrate in vivo in liver structures [76,77]. In media containing nicotinamide, HGF, activin-A and pentagastrn, ADSC spheroids could be induced to pancreatic islet-like cells that could produce insulin somatostatin and glucagon [78]. Cultured in serum-free neurobasal media containing epitelial growth factor (EGF) and FGF, ADSC spheroids could be converted to „neurospheres” and induced to differentiate to neurons [79] and peripheral glial cells [80].

ADSCs could be differentiated as well to Schwann-like cells having similar features and function to primary Schwann cells in vitro and in vivo. This could support the application of autologus transplantation of these cells for treating spinal cord [81] or peripheral nerve injuries [82]. ADSCs were as well differentiated to neurotrophic factor secreting cells (NTF-SCs) that could be transplanted or induced to migrate towards neural lesions aiming to treat neurodegenerative diseases such as Hungtington's [83] or Parkinson's disease [84]. Short-time culture of ADSCs as spheroids in serum-free media after longer time (6 passages) culture in monolayer was able to increase clonogenicity and differentiation potential to neural lineages by increasing the level of miRNAs involved in multipotency and differentiation. Conversion to $3 \mathrm{D}$ culture could be of interest for improving cell manufacturing protocols for treating neurodegenerative diseases [61].

Spheroid culture of MSCs prevents replicative senescence and contributes to preservation of stemness as expressed by colony formation potential [85], supporting the usefulness of this culture system for clinical applications of MSC-based therapies.

Human BMMSCs grown as spheroids were found to be more effective than 2D-cultured populations in suppressing inflammatory responses in co-culture with lipopolyssacharide (LPS) activated macrophages and in a mouse model of peritonitis. BMMSCs spheroid displayed enhanced expression of anti-inflammatory proteins TNF- $a$ stimulated gene/protein 6 (TSG-6) and stanniocalcin-1 (STC-1) as well as anticancer proteins IL-24, TNF- $\alpha$-related apoptosis inducing ligand, and CD82. Moreover, in the same study, cells originating from spheroid culture had one-fourth of the volume of $2 \mathrm{D}$-cultivated cells this possibly explains their higher lung trafficking potential and increased organ distribution after intravenous delivery [86]. Increased levels of prostaglandin E2 (PGE2) were found in conditioned medium (CM) from MSCs spheroid compared to human fibroblast spheroids Spheroid MSC CM was efficient in preventing LPS activated macrophages from secreting pro-inflammatory cytokines TNF- $\alpha$, CXCL2, IL6, IL12p40, and IL23 [87]. 2D-culture-expanded MSCs are shown to be activated by spheroid formation to produce anti-inflammatory factors (TSG-6, STC-1, and PGE2) for potential use for clinical applications in immune modulation [88].

However, it is to be noted that the use of serum-free media formulations do not result in MSCs spheroids with anti-inflammatory properties [33]. The cause of this variability is yet to be elucidated as it is crucial for the design of xenofree clinical grade cells and for standardization of manufacturing protocols [89].

CM collected from human MCSc cultivated as spheroids were more effective than CM from adherent cultures in stimulating human umbilical vein endothelial cells (HUVECs) proliferation, migration, and basement membrane invasion. The VEGF and angiogenin mediated in vitro angiogenetic effect on HUVECs showed little variation when MSCs shperoids were cultivated in serum-free conditions [59]. Increased release of angiogenetic cytokines and pro-angiogenetic growth factors such as FGF2 or HGF by MSCs spheroids [90] could explain the in vivo potential for tissue repair and revascularization. Thus, in a mice model of hindlimb ischemia, direct transplantation of human cord blood mesenchymal stem cells (hCBMSCs) significantly increased the number of microvessels and smooth $\alpha$-actin positive vasculature compared to untreated controls [91]. Enhanced angiogenesis and faster healing as well as significantly increased cell engraftment of spheroid precultivated ADSCs compared to $2 \mathrm{D}$ counterparts was reported in a mouse model of unhealing skin wound [92].

As oposed to monolayer expanded cells, MSCs from 3D culture did not produce embolism after intra carotidian delivery in a rat model with stroke. Improved reduction of cerebral infarctation size $(70 \%)$ was reported in this study, as well as evidence of direct contribution of injected cells to newly formed neurons and increased presence of endogenous glial fibrillary acidic protein-positive (GFAP) neural progenitors surrouding the infactation zone [93].

Substantial decrease of MSCs spheroids entrapped in lungs after intravenous delivery by reduced integrin ( $\beta 1$, integrin $\alpha 5$, or integrins $\alpha \mathrm{V} \beta 3$ ) expression compared to monolayer cultivated cells [87] could recommend their use for systemic delivery of cell therapies.

Taken together, evidence exist for improved cell survival after delivery, enhanced engraftment, pro-angiogenetic cytokine release and tissue regeneration potential of MSCs spheroids. However, controversial reports ask for better understanding of self aggregation phenomena and its influence on cell phenotype. Thus, 3D-cultured 
MSCs were found to generate local microgliosis, decreased tyrosine hydroxylase (TH) levels and reduced new cell formation in substantia nigra of a rat model of Parkinson's disease failing to improve motor neuron functions compared to monolayer cultivated cells [94]. The influences of aggregate culture on cell characteristics especially after in vivo transplantation should be therefore carefully assessed in respect to a particular clinical application. It should also be noted that cellular self-formed aggregates from one side; do not fully reporduce the complexity of a tissue and could introduce cell phenotype that is incompletelly known, from the other side. Functional particularities of such cells both in vitro and after implantation in vivo needs to be better understood before forwarding their use to clinical applications.

\section{Conclusion}

Three dimensional cell cultures allows for enhanced reproduction of complex intercellular and cell-ECM communication, more closely reproducing the natural environment. Increasing numbers of studies are reporting the superiority of MSCs biological features, enhanced cell survival after transplantation as well as higher impact on tissue regeneration.

However, complete genetic and epigenetic profiling of MSCs from various sources needs to be performed in order to enable prediction of their behavior after implantation. This will allow the elucidation of important questions about modified cell biology in this particular, artificially created state. Suspension culture of mesenchymal cells that are in normal condition anchorage dependent and adoption of spherical shape could induce a rearrangement of cytoskeleton, potentially involved in regaining in earlier stages of potency via a mechanism E-cadherin mediated. While this is a hypothesis that needs to be confirmed, it would require further attention. The influence of cytoskeleton changes the responsibility of the modified cell shape and physical properties of cell stemness need to be better understood before using these cells as therapeutic agents. Manipulation of cell population with non-defined reacquired pluripotency can be hazardous and any potential of tumorigenesis has to be clearly ruled out. The molecular basis of spheroid variability in relation to culture media changes, especially the presence of serum, is another aspect that needs to be more scrutinized. Comparative studies of serum and serum-free cytokine release and differentiation potential of MSCs spheroids are needed to clarify their therapeutic role and to scale up cell manufacturing for clinical application.

\section{References}

1. Friedenstein AJ, Gorskaja JF, Kulagina NN (1976) Fibroblast precursors in normal and irradiated mouse hematopoietic organs. Exp Hematol 4: 267-274.

2. Owen M, Friedenstein AJ (1988) Stromal stem cells: marrow-derived osteogenic precursors. Ciba Found Symp 136: 42-60.

3. Friedenstein AJ, Chailakhyan RK, Gerasimov UV (1987) Bone marrow osteogenic stem cells: in vitro cultivation and transplantation in diffusion chambers. Cell Tissue Kinet 20: 263-272.

4. Zuk PA, Zhu M, Mizuno H, Huang J, Futrell JW, et al. (2001) Multilineage cells from human adipose tissue: implications for cell-based therapies. Tissue Eng 7: 211-228.

5. Chen JC, Goldhamer DJ (2003) Skeletal muscle stem cells. Reprod Biol Endocrinol 1: 101.

6. Song L, Young NJ, Webb NE, Tuan RS (2005) Origin and characterization of multipotential mesenchymal stem cells derived from adult human trabecular bone. Stem Cells Dev 14: 712-721.
7. Huang GT, Yamaza T, Shea LD, Djouad F, Kuhn NZ, et al. (2010) Stem Progenitor cell-mediated de novo regeneration of dental pulp with newly deposited continuous layer of dentin in an in vivo model. Tissue Eng Part A 16: $605-615$

8. Erices A, Conget $\mathrm{P}$, Minguell JJ (2000) Mesenchymal progenitor cells in human umbilical cord blood. Br J Haematol 109: 235-242.

9. Caplan Al (1994) The mesengenic process. Clin Plast Surg 21: 429-435

10. Caplan Al (2007) Adult mesenchymal stem cells for tissue engineering versus regenerative medicine. J Cell Physiol 213: 341-347.

11. Trzaska KA, Rameshwar P (2011) Dopaminergic neuronal differentiation protocol for human mesenchymal stem cells. Methods Mol Biol 698: 295-303.

12. Radtke C, Schmitz B, Spies M, Kocsis JD, Vogt PM (2009) Peripheral glial cell differentiation from neurospheres derived from adipose mesenchymal stem cells. Int J Dev Neurosci 27: 817-823.

13. Aurich H, Sgodda M, Kaltwasser P, Vetter M, Weise A, et al. (2009) Hepatocyte differentiation of mesenchymal stem cells from human adipose tissue in vitro promotes hepatic integration in vivo. Gut 58: 570-581.

14. Oswald J, Boxberger S, Jørgensen B, Feldmann S, Ehninger G, et al. (2004) Mesenchymal stem cells can be differentiated into endothelial cells in vitro. Stem Cells 22: 377-384.

15. Barry FP, Murphy JM, O'Brien T, Mahon B (2005) Mesenchymal stem cell transplantation for tissue repair. Semin Plast Surg 19: 229-239.

16. Dominici M, Le Blanc K, Mueller I, Slaper-Cortenbach I, Marini F, et al. (2006) Minimal criteria for defining multipotent mesenchymal stromal cells. The International Society for Cellular Therapy position statement. Cytotherapy 8: 315-317.

17. Alimperti S, Lei P, Wen Y, Tian J, Campbell AM, et al. (2014) Serum-free spheroid suspension culture maintains mesenchymal stem cell proliferation and differentiation potential. Biotechnol Prog 30: 974-983.

18. Yamaguchi Y, Ohno J, Sato A, Kido H, Fukushima T (2014) Mesenchymal stem cell spheroids exhibit enhanced in-vitro and in-vivo osteoregenerative potential. BMC Biotechnol 14: 105.

19. Bourin P, Bunnell BA, Casteilla L, Dominici M, Katz AJ, et al. (2013) Stromal cells from the adipose tissue-derived stromal vascular fraction and culture expanded adipose tissue-derived stromal/stem cells: a joint statement of the International Federation for Adipose Therapeutics and Science (IFATS) and the International Society for Cellular Therapy (ISCT). Cytotherapy 15: 641648.

20. Pittenger MF, Mackay AM, Beck SC, Jaiswal RK, Douglas R, et al. (1999) Multilineage potential of adult human mesenchymal stem cells. Science 284 : 143-147.

21. Thomas R, Ratcliffe E (2012) Automated adherent human cell culture (mesenchymal stem cells). Methods Mol Biol 806: 393-406.

22. Lechanteur C, Baila S, Janssens ME, Giet O, Brique A, et al. (2014) Largescale clinical expansion of mesenchymal stem cells in the gmp-compliant, closed automated quantum ${ }^{\circledR}$ cell expansion system: comparison with expansion in traditional t-flasks. J Stem Cell Res Ther 4: 1-11.

23. Bieback K (2013) Platelet lysate as replacement for fetal bovine serum in mesenchymal stromal cell cultures. Transfus Med Hemother 40: 326-335.

24. Page H, Flood P, Reynaud EG (2013) Three-dimensional tissue cultures: current trends and beyond. Cell Tissue Res 352: 123-131.

25. Bork S, Pfister S, Witt H, Horn P, Korn B, et al. (2010) DNA methylation pattern changes upon long-term culture and aging of human mesenchymal stromal cells. Aging Cell 9: 54-63.

26. Stolzing A, Coleman N, Scutt A (2006) Glucose-induced replicative senescence in mesenchymal stem cells. Rejuvenation Res 9: 31-35.

27. Saleh FA, Frith JE, Lee JA, Genever PG (2012) Three-dimensional in vitro culture techniques for mesenchymal stem cells. Methods Mol Biol 916: 31-45.

28. Brown PT, Handorf AM, Jeon WB, Li WJ (2013) Stem cell-based tissue 
engineering approaches for musculoskeletal regeneration. Curr Pharm Des 19: 3429-3445

29. Bogdanova-Jatniece A, Berzins U, Kozlovska T (2014) Growth properties and pluripotency marker expression of spontaneously formed three-dimensional aggregates of human adipose-derived stem cells. Int J Stem Cells 7: 143-

30. Mueller-Klieser W (1987) Multicellular spheroids. A review on cellular aggregates in cancer research. J Cancer Res Clin oncol 113: 101-122.

31. Sasai Y (2013) Cytosystems dynamics in self-organization of tissue architecture. Nature 493: 318-326.

32. Fennema E, Rivron N, Rouwkema J, van Blitterswijk C, de Boer J (2013) Spheroid culture as a tool for creating 3D complex tissues. Trends Biotechnol 31: 108-115.

33. Cesarz Z, Tamama K (2015) Spheroid culture of mesenchymal stem cells Stem Cells Int (In Press).

34. Foty R (2011) A simple hanging drop cell culture protocol for generation of 3D spheroids. J Vis Exp 6: 2720

35. Metzger W, Sossong D, Bächle A, Pütz N, Wennemuth G, et al. (2011) The liquid overlay technique is the key to formation of co-culture spheroids consisting of primary osteoblasts, fibroblasts and endothelial cells. Cytotherapy 13: 1000-1012

36. Chan HF, Zhang Y, Ho YP, Chiu YL, Jung Y, et al. (2013) Rapid formation of multicellular spheroids in double-emulsion droplets with controllable microenvironment. Sci Rep 3: 3462.

37. Okubo H, Matsushita M, Kamachi H, Kawai T, Takahashi M, et al. (2002) A novel method for faster formation of rat liver cell spheroids. Artif Organs 26 : 497-505.

38. Achilli TM, Meyer J, Morgan JR (2012) Advances in the formation, use and understanding of multi-cellular spheroids. Expert Opin Biol Ther 12: 1347 1360 .

39. Martin GR (1981) Isolation of a pluripotent cell line from early mouse embryos cultured in medium conditioned by teratocarcinoma stem cells. Proc Nat Acad Sci U S A 78: 7634-7638

40. Barry F, Boynton RE, Liu B, Murphy JM (2001) Chondrogenic differentiation of mesenchymal stem cells from bone marrow: differentiation-dependen gene expression of matrix components. Exp Cell Res 268: 189-200.

41. Daquinag AC, Souza GR, Kolonin MG (2013) Adipose tissue engineering in three-dimensional levitation tissue culture system based on magnetic nanoparticles. Tissue Eng Part C Methods 19: 336-344.

42. Liu J, Kuznetsova LA, Edwards GO, Xu J, Ma M, et al. (2007) Functiona three-dimensional HepG2 aggregate cultures generated from an ultrasound trap: comparison with HepG2 spheroids. J Cell Biochem 102: 1180-1189.

43. Duguay D, Foty RA, Steinberg MS (2003) Cadherin-mediated cell adhesion and tissue segregation: qualitative and quantitative determinants. Dev Biol 253: 309-323.

44. Foty RA, Pfleger CM, Forgacs G, Steinberg MS (1996) Surface tensions of embryonic tissues predict their mutual envelopment behavior. Development 122: $1611-1620$

45. Delaine-Smith RM, Reilly GC (2012) Mesenchymal stem cell responses to mechanical stimuli. Muscles Ligaments Tendons J 2: 169-180.

46. Shevde NK, Mael AA (2013) Techniques in embryoid body formation from human pluripotent stem cells. Methods Mol Biol 946: 535-546.

47. Vallier L, Pedersen RA (2005) Human embryonic stem cells: an in vitro model to study mechanisms controlling pluripotency in early mammalian development. Stem Cell Rev 1: 119-130.

48. Reynolds BA, Rietze RL (2005) Neural stem cells and neurospheres--reevaluating the relationship. Nat Methods 2: 333-336.

49. Dontu G, Abdallah WM, Foley JM, Jackson KW, Clarke MF, et al. (2003)
In vitro propagation and transcriptional profiling of human-mammary stem/ progenitor cells. Genes Dev 17: 1253-1270.

50. Li H, Dai Y, Shu J, Yu R, Guo Y, et al. (2015) Spheroid cultures promote the stemness of corneal stromal cells. Tissue Cell 47: 39-48.

51. Lin RZ, Chang HY (2008) Recent advances in three-dimensional multicellular spheroid culture for biomedical research. Biotechnol J 3: 1172-1184.

52. Subramanian K, Owens DJ, O'Brien TD, Verfaillie CM, Hu WS (2011) Enhanced differentiation of adult bone marrow-derived stem cells to liver lineage in aggregate culture. Tissue Eng Part A 17: 2331-2341.

53. Okumura K, Nakamura K, Hisatomi Y, Nagano K, Tanaka Y, et al. (2003) Salivary gland progenitor cells induced by duct ligation differentiate into hepatic and pancreatic lineages. Hepatology 38: 104-113.

54. Watanabe K, Kamiya D, Nishiyama A, Katayama T, Nozaki S, et al. (2005) Directed differentiation of telencephalic precursors from embryonic stem cells. Nat Neurosc 8: 288-296.

55. Sasai $Y$ (2013) Next-generation regenerative medicine: organogenesis from stem cells in 3D culture. Cell Stem Cell 12: 520-530.

56. Eiraku M, Takata N, Ishibashi H, Kawada M, Sakakura E, et al. (2011) Selforganizing optic-cup morphogenesis in three-dimensional culture. Nature 472: $51-56$

57. Paşca AM, Sloan SA, Clarke LE, Tian Y, Makinson CD, et al. (2015) Functional cortical neurons and astrocytes from human pluripotent stem cells in 3D culture. Nat Method 12: 671-678.

58. Sasai Y (2013) Cytosystems dynamics in self-organization of tissue architecture. Nature 493: 318-326.

59. Lin RZ, Chang HY (2008) Recent advances in three-dimensional multicellular spheroid culture for biomedical research. Biotechnol J 3: 1172-1184.

60. Tsai AC, Liu Y, Yuan X, Ma T (2015) Compaction, fusion, and functiona activation of three-dimensional human mesenchymal stem cell aggregate. Tissue Eng Part A 21: 1705-1719.

61. Zhang Q, Nguyen AL, Shi S, Hill C, Wilder-Smith P, et al. (2012) Threedimensional spheroid culture of human gingiva-derived mesenchymal stem cells enhances mitigation of chemotherapy-induced oral mucositis. Stem Cells Dev 21: 937-947.

62. Baraniak PR, Cooke MT, Saeed R, Kinney MA, Fridley KM, et al. (2012) Stiffening of human mesenchymal stem cell spheroid microenvironments induced by incorporation of gelatin microparticles. J Mech Behav Biomed Mater 11: 63-71.

63. Lee J, Abdeen AA, Kilian K (2014) Rewiring mesenchymal stem cell lineage specification by switching the biophysical microenvironment. Sci Rep 4: 5188

64. Baraniak PR, McDevitt TC (2012) Scaffold-free culture of mesenchymal stem cell spheroids in suspension preserves multilineage potential. Cell Tissue Res 347: 701-711.

65. Potapova IA, Gaudette GR, Brink PR, Robinson RB, Rosen MR, et al. (2007) Mesenchymal stem cells support migration, extracellular matrix invasion, proliferation, and survival of endothelial cells in vitro. Stem Cells 25: 17611768.

66. Yeh HY, Liu BH, Sieber M, Hsu SH (2014) Substrate-dependent gene regulation of self-assembled human MSC spheroids on chitosan membranes. BMC Genomics 15: 10.

67. Guo L, Zhou Y, Wang S, Wu Y (2014) Epigenetic changes of mesenchymal stem cells in three-dimensional (3D) spheroids. J Cell Mol Med 18: 2009 2019.

68. Xu N, Papagiannakopoulos T, Pan G, Thomson JA, Kosik KS, et al. (2009) MicroRNA-145 regulates OCT4, SOX2, and KLF4 and represses pluripotency in human embryonic stem cells. Cell 137: 647-658.

69. Le Blanc K, Götherström C, Ringdén O, Hassan M, McMahon R, et al. (2005) Fetal mesenchymal stem-cell engraftment in bone after in utero transplantation in a patient with severe osteogenesis imperfecta. Transplantation 79: 16071614. 
70. Henning TD, Gawande R, Khurana A, Tavri S, Mandrussow L, et al. (2012) Magnetic resonance imaging of ferumoxide-labeled mesenchymal stem cells in cartilage defects: in vitro and in vivo investigations. Mol Imaging 11: 197209.

71. Tolar J, Le Blanc K, Keating A, Blazar BR (2010) Concise review: hitting the right spot with mesenchymal stromal cells. Stem Cells 28: 1446-1455.

72. Le Blanc K (2003) Immunomodulatory effects of fetal and adult mesenchymal stem cells. Cytotherapy 5: 485-489.

73. Yamaguchi Y, Ono J, Sato A, Kido H, Fukushima T (2014) Mesenchymal stem cells exhibit enhanced in vitro and in vivo osteoregenerative potential. BMC Biotechnol 14: 105.

74. Kapur SK, Wang X, Shang H, Yun S, Li X, et al. (2012) Human adipose stem cells maintain proliferative, synthetic and multipotential properties when suspension cultured as self-assembling spheroids. Biofabrication 4: 025004.

75. Shiffman MA, Alberto GD, Franco B (2014) Stem cells in aesthetic procedures Springer.

76. No da Y, Lee SA, Choi YY, Park D, Jang JY, et al. (2012) Functional 3D human primary hepatocyte spheroids made by co-culturing hepatocytes from partial hepatectomy specimens and human adipose-derived stem cells. PLoS One 7: e50723.

77. Timper K, Seboek D, Eberhardt M, Linscheid P, Christ-Crain M, et al. (2006) Human adipose tissue-derived mesenchymal stem cells differentiate into insulin, somatostatin, and glucagon expressing cells. Biochem Biophys Res Commun 341: 1135-1140.

78. Yu JM, Bunnell BA, Kang SK (2011) Neural differentiation of human adipose tissue-derived stem cells. Methods Mol Biol 702: 219-231.

79. Radtke C, Schmitz B, Spies M, Kocsis JD, Vogt PM (2009) Peripheral glia cell differentiation from neurospheres derived from adipose mesenchyma stem cells. Int J Dev Neurosci 27: 817-823.

80. Ghasemi N, Razavi S (2014) Transdifferentiation potential of adipose-derived stem cells into neural lineage and their application. J Histol Histopathol 1: 12.

81. Kaewkhaw R, Scutt AM, Haycock JW (2011) Anatomical site influences the differentiation of adipose-derived stem cells for schwann-cell phenotype and function. Glia 59: 734-749.

82. Sadan O, Shemesh N, Barzilay R, Dadon-Nahum M, Blumenfeld-Katzir T, et al. (2012) Mesenchymal stem cells induced to secrete neurotrophic factors attenuate quinolinic acid toxicity: a potential therapy for Huntington's disease. Exp Neurol 234: 417-427.

83. Sandan O, Bahat-Stromza M, Barhum Y, Levy YS, Pisnevsky A, et al. (2009)
Protective effects of neurotrophic factor-secreting cells in a 6-OHDA rat model of Parkinson disease. Stem Cells Dev 18: 1179-1190.

84. Pochampally R (2008) Colony forming unit assays for MSCs. Methods Mol Biol 449: 83-91.

85. Bartosh TJ, Ylöstalo JH, Mohammadipoor A, Bazhanov N, Coble K (2010) Aggregation of human mesenchymal stromal cells (MSCs) into 3D spheroids enhances their antiinflammatory properties. Proc Natl Acad Sci U S A 107: 13724-13729.

86. Ylöstalo JH, Bartosh TJ, Coble K, Prockop DJ (2012) Human mesenchymal stem/stromal cells cultured as spheroids are self-activated to produce prostaglandin E2 that directs stimulated macrophages into an antiinflammatory phenotype. Stem Cells 30: 2283-2296.

87. Bartosh TJ, Ylostalo JH (2014) Preparation of anti-inflammatory mesenchymal stem/precursor cells (MSCs) through sphere formation using hanging-drop culture technique. Curr Protoc Stem Cell Biol 28: Unit 2B.6.

88. Menard C, Pacelli L, Bassi G, Dulong J, Bifari F (2013) Clinical-grade mesenchymal stromal cells produced under various good manufacturing practice processes differ in their immunomodulatory properties: standardization of immune quality controls. Stem Cells Dev 22: 1789-1801.

89. Potapova IA, Brink PR, Cohen IS, Doronin SV (2008) Culturing of human mesenchymal stem cells as three-dimensional aggregates induces functional expression of CXCR4 that regulates adhesion to endothelial cells. J Biol Chem 283: 13100-13107

90. Bhang SH, Lee S, Shin JY, Lee TJ, Kim BS (2012) Transplantation of cord blood mesenchymal stem cells as spheroids enhances vascularization. Tissue Eng Part A 18: 2138-2147.

91. Cheng NC, Chen SY, Li JR, Young TH (2013) Short-term spheroid formation enhances the regenerative capacity of adipose-derived stem cells by promoting stemness, angiogenesis, and chemotaxis. Stem Cells Transl Med 2: 584-594.

92. Guo L, Ge J, Zhou Y, Wang S, Zhao RC, et al. (2014) Three-dimensional spheroid-cultured mesenchymal stem cells devoid of embolism attenuate brain stroke injury after intra-arterial injection. Stem Cells Dev 23: 978-989.

93. Wang S, Guo L, Ge J, Yu L, Cai T, et al. (2015) Excess integrins cause lung entrapment of mesenchymal stem cells. Stem Cells [Epub ahead of print].

94. Berg J, Roch M, Altschüler J, Winter C, Schwerk A, et al. (2015) Human adipose-derived mesenchymal stem cells improve motor functions and are neuroprotective in the 6-hydroxydopamine-rat model for Parkinson's disease when cultured in monolayer cultures but suppress hippocampal neurogenesis and hippocampal memory function when cultured in spheroids. Stem Cell Rev 11: 133-149.

\section{Acknowledgements}

The author thanks the reviewers of the manuscript for their scientific support. 